\title{
Primordial Star Formation in the First Galaxies
}

\author{
Chalence Safranek-Shrader*, Meghann Agarwal, Miloš Milosavljević, and Volker \\ Bromm \\ Department of Astronomy and Texas Cosmology Center, The University of Texas at Austin \\ E-mail: ctsseastro.as.utexas.edu
}

\begin{abstract}
We present results of a high-resolution cosmological simulation which explores the process of primordial gas collapsing into a high-redshift atomic cooling halo, typically considered to be a 'first galaxy'. We consider a physically motivated scenario where a strong molecule destroying Lyman-Werner (LW) background prevents gaseous collapse, and thus star formation, in small mass halos. Only when molecular hydrogen can shield itself from LW radiation in a sufficiently massive halo will star formation be possible. We find that in a $1 \mathrm{Mpc}^{3}$ (comoving) box with a $\mathrm{LW}$ background corresponding to $J_{21}=100$ collapse first occurs in a $3 \times 10^{7} M_{\odot}$ dark matter halo at $z \simeq 12.1$. A distinct phase transition occurs in the center of the halo when molecular hydrogen $\left(\mathrm{H}_{2}\right)$ begins to self-shield, allowing baryons to cool, and to form a supersonically turbulent $\sim 10 \mathrm{pc}$ core. We investigate the character of turbulence in this core, fragmentation properties, and impact on next generation star formation.
\end{abstract}

Frank N. Bash Symposium New Horizons In Astronomy, October 9-11, 2011

Austin Texas

\footnotetext{
${ }^{1}$ University of Texas at Austin \& Texas Cosmology Center * Speaker.
} 


\section{Introduction}

The first metal-free stars (Pop. III) are theorized to form in $\sim 10^{6} \mathrm{M}_{\odot}$ dark matter minihalos at $z \sim 20-50$ (e.g., [1]). Determining their detailed initial mass function (IMF) is an ongoing challenge - estimates place their characteristic mass somewhere between $\sim 10$ and $\sim 100 M_{\odot}$. The detectability of individual, high-redshift Pop. III stars is extremely low, even with the upcoming James Webb Space Telescope. However, clusters of Pop. III stars (which may form in larger mass halos if gas can remain metal-free) may be detectable [2]. Here, we present a cosmological simulation focused on the collapse of metal-free gas into the host of a typical first galaxy - an atomic cooling halo. We assume this halo forms in a region of space with a strong background LW radiation field capable of photodissociating $\mathrm{H}_{2}$ and preventing gaseous collapse into low mass halos. Only when a halo becomes massive enough that $\mathrm{H}_{2}$ can begin to self-shield will collapse, and thus star formation, be possible. We are primarily interested in this transition. When and where does this self-shielding transition take place? What environment does it produce? And what kind of subsequent star formation can we expect?

\section{Methodology and Physical Details}

We use the adaptive mesh refinement (AMR) code FLASH [3] to conduct our simulation. FLASH solves the Eulerian equations of hydrodynamics in comoving coordinates on an spatially adaptive grid. Baryons are represented by a hydrodynamic fluid while dark matter is modeled by collisionless, massive particles. The gravitational potential is computed by an iterative multigrid Poisson solver. We initialize our simulation at $z=145$ in a $1 \mathrm{Mpc}^{3}$ (comoving) box. Cosmological initial conditions were generated using current cosmological parameters. Our chemical model evolves the most thermally and chemically important species in metal-free gas $-\mathrm{H}, \mathrm{H}^{-}, \mathrm{H}^{+}$, $\mathrm{e}^{-}, \mathrm{H}_{2}, \mathrm{H}_{2}^{+}, \mathrm{He}, \mathrm{He}^{+}, \mathrm{He}^{++}, \mathrm{D}, \mathrm{D}^{+}$, and HD. To trigger AMR refinement, we use two separate criteria based on local gas properties: one dependent on gas overdensity, and another enforcing that the Jeans length, $L_{\mathrm{J}}=\left(\pi c_{s}^{2} / G \rho_{g}\right)^{1 / 2}$, is adequately resolved by at least 12 grid cells. The most significant cooling mechanisms in metal-free first galaxies are Ly $\alpha$ emission from neutral hydrogen, molecular hydrogen cooling, and HD cooling. Ly $\alpha$ cooling is an extremely effective coolant above $\sim 10^{4} \mathrm{~K}$ and can cool gas to $T \sim 8000 \mathrm{~K}$. Ro-vibrational emission from molecular hydrogen can potentially cool the gas further, although not below $\sim 200 \mathrm{~K}$. HD, if present, can cool gas further (limited by the CMB temperature floor), although an elevated electron fraction is likely required for it to form in significant quantities.

\section{Pathway to a Metal Free Galaxy}

For gas in our target halo to remain metal free, star formation in minihalos must be suppressed (e.g., [4], [5]). To this end, we impose a FUV flux of $J_{21}=100$ across the entire box, such that $J_{v}=J_{21} 10^{-21} \mathrm{erg} \mathrm{s}^{-1} \mathrm{~cm}^{-2} \mathrm{~Hz}^{-1} \mathrm{sr}^{-1}$ at the Lyman-limit. Above the Lyman-limit, $J_{v}=0$. This UV flux strength will prevent gaseous collapse into minihalos (e.g., [6]) as it destroys molecular hydrogen, the key cooling agent for the first stars. From this radiation field, we only consider the photodissociation of $\mathrm{H}_{2}$ and $\mathrm{HD}$ (not, for example, $\mathrm{H}^{-}$destruction). We track the $\mathrm{H}_{2}$ and $\mathrm{HD}$ 
column densities ( $N_{\mathrm{H}_{2}}$ and $\left.N_{\mathrm{HD}}\right)$ self-consistently to each point in the box assuming radiation is incident from the sides of the box. Given these column densities we can write the photo-dissociation rate of $\mathrm{H}_{2}$ as $k_{\mathrm{H}_{2}}=1.38 \times 10^{9} J_{\mathrm{LW}} f_{\text {shield }}^{\mathrm{H}_{2}}$, where $J_{\mathrm{LW}}$ is the mean radiation intensity at $h v=12.87 \mathrm{eV}$ and $f_{\text {shield }}^{\mathrm{H}_{2}}$ accounts for $\mathrm{H}_{2}$ self-shielding, given by [7]

$$
f_{\text {shield }}^{\mathrm{H}_{2}}=\frac{0.965}{\left(1+x / b_{5}\right)^{1.1}}+\frac{0.035}{(1+x)^{0.5}} \times \exp \left[-8.5 \times 10^{-4}(1+x)^{0.5}\right]
$$

where $x=N_{\mathrm{H}_{2}} / 5 \times 10^{14} \mathrm{~cm}^{-2}, b_{5}=b / 10^{5} \mathrm{~cm} \mathrm{~s}^{-1}$, and $b$ is the velocity spread parameter of the gas. A similar approach is used for HD.

\section{Results}

With $J_{21}=100, \mathrm{H}_{2}$ is vigorously destroyed and gas density can only increase adiabatically. By $z \simeq 13$, a halo has grown massive enough that its virial temperature is on the order of $T \sim 10^{4} \mathrm{~K}$, the atomic cooling threshold, and its central region begins to collapse roughly isothermally at $T \simeq 8000 \mathrm{~K}$. With increasing density, the $\mathrm{H}_{2}$ formation and cooling rates increase. Additionally, the $\mathrm{H}_{2}$ column rises, increasing the degree of LW self-shielding, and decreasing the $\mathrm{H}_{2}$ destruction rate. These effects amplify each other and cause the $\mathrm{H}_{2}$ abundance to increase in a runaway fashion. Specifically, this process occurs at a $J_{21}$-dependent critical density when $t_{\text {cool, } \mathrm{H}_{2}}<t_{\mathrm{ff}}$ (e.g., [8]) causing the gas to cool rapidly.
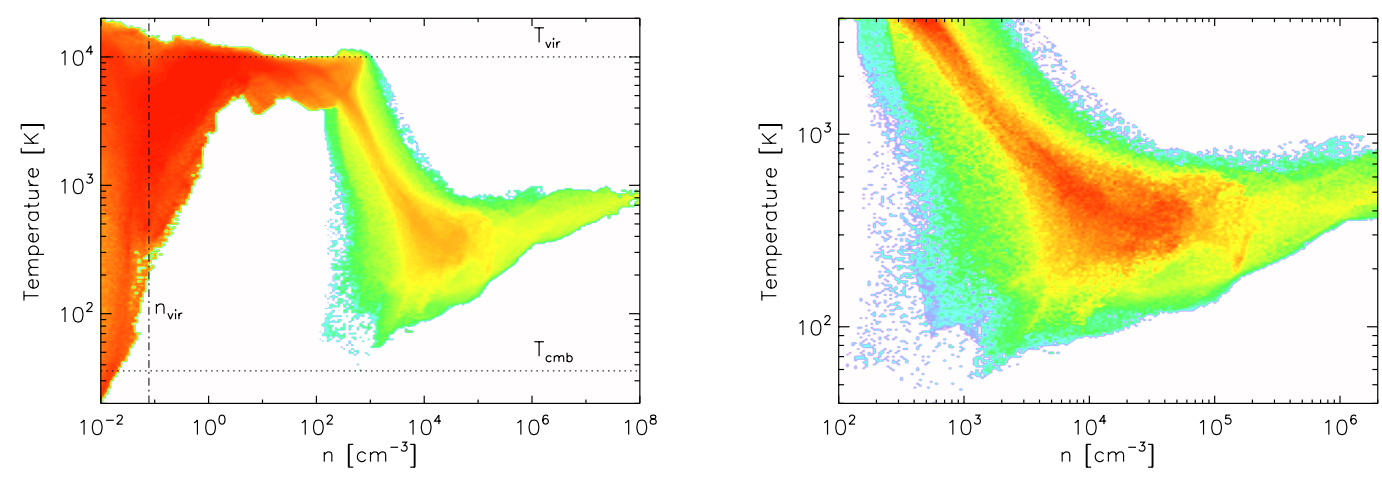

Figure 1: Left: Density-temperature phase plot of gas within $2 R_{\mathrm{vir}}$ of the target halo at $z \simeq 12.1$. We display the virial temperature of the halo, CMB temperature, and $n_{\mathrm{vir}}=18 \pi^{2} \bar{n}$. Right: Same as the right figure, but different axes ranges, illustrating the 'loitering state' at $n \sim 2 \times 10^{4} \mathrm{~cm}^{-3}$ and $T \sim 400 \mathrm{~K}$ and the further evolution along the standard metal-free molecular cooling track. Color coding corresponds to mass.

In Fig. 1 we show density-temperature phase plots at $z=12.2$. Fig. 1(a), with a large dynamic range, illustrates gas being shock heated to $T \simeq 10^{4} \mathrm{~K}$ and the subsequent isothermal collapse occurring to a density of $n \sim 10^{2} \mathrm{~cm}^{-3}$. Fig. 1(b) highlights the state of the gas at intermediate densities. This behavior is similar in many respects to the canonical picture of metal-free gas collapse into a minihalo, as there is a fairly well defined 'loitering state' of gas at $n \sim 2 \times 10^{4} \mathrm{~cm}^{-3}$ and $T \sim 400 \mathrm{~K}$, corresponding to the point where the energy levels of $\mathrm{H}_{2}$ reach LTE and its cooling rate decreases. Further collapse occurs along the well-studied metal-free molecular cooling track. When the gas density reaches $n=10^{8} \mathrm{~cm}^{-3}$, the self-shielding core (defined such that $f_{\text {shield }}^{\mathrm{H}_{2}}<$ 
$10^{-2}$ ) has a mass of $\simeq 1.1 \times 10^{4} \mathrm{M}_{\odot}$, a diameter $\sim 10 \mathrm{pc}$, and mass- and volume-weighted RMS mach numbers $\left(\mathrm{Ma} \equiv c_{\mathrm{s}} / v_{\text {tot }}\right)$ of 3.6 and 3.3, respectively. If this core produces one burst of star formation, the maximum star formation efficiency with respect to the total baryonic mass in the halo is $f_{*}=M_{*} / M_{b a r}=2 \times 10^{-3}$, likely much lower given we do not model any sort of stellar feedback. We introduce sink particles, using the method of [9], at a density of $n=10^{8} \mathrm{~cm}^{-3}$. For at least $2.5 \times 10^{4} \mathrm{yrs}$ after the formation of this sink no others are created, implying additional gaseous fragmentation and collapse (if any) in this timeframe occurred within the sink particle, an unresolved region. In Fig. 2(a), we plot the mass- and volume-weighted density PDFs for the self-shielding core. At low densities, the PDFs are well fit by log-normal distributions, suggesting supersonic turbulence, in the form of complex, interacting shocks, may be responsible for shaping the overall density distribution (e.g., [10]). The PDF power law tail at high densities is expected, arising from the gas gravitationally decoupling from the turbulent motion. Fig. 2(a) also displays standard deviation $\sigma$ of the log-normal fits, which can be related to the mach number as $\sigma=$ $\ln \left(1+b^{2} \mathrm{Ma}^{2}\right)^{1 / 2}$, where numerical experiments have found $b$ to lie between 0.2 and 0.5 . The lognormal fits then correspond to $\mathrm{Ma}=1.71 / \mathrm{b}$ and $\mathrm{Ma}=1.29 / \mathrm{b}$ for the mass- and volume-weighting, respectively, very much consistent with the measured mach numbers in the self-shielding core.
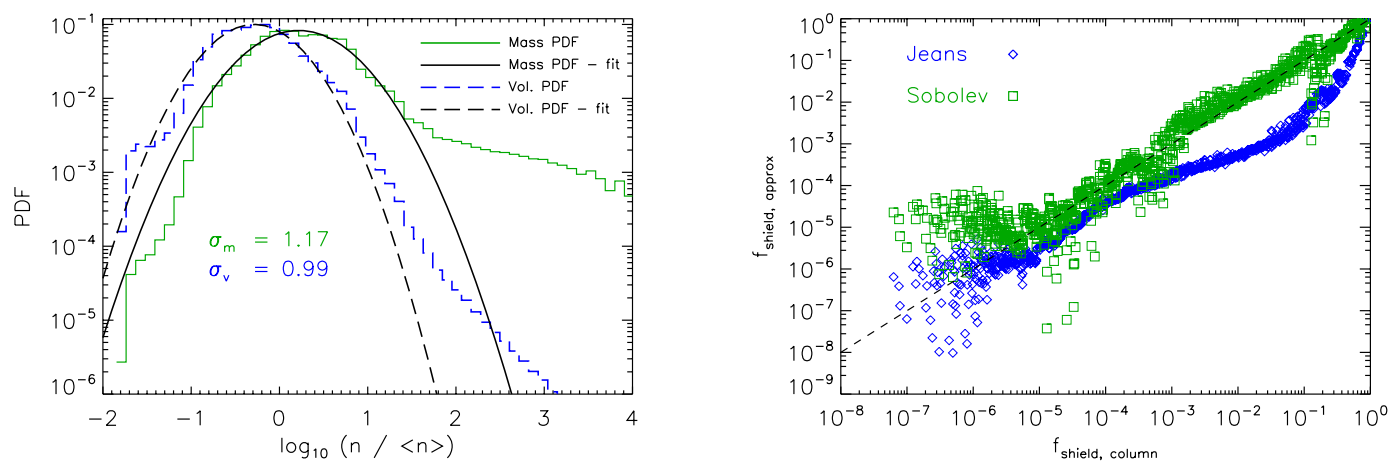

Figure 2: Left: Mass-weighted (green histogram) and volume-weighted (blue dashed histogram) density PDFs of self-shielding gas $\left(f_{\text {shield }}^{\mathrm{H}_{2}}<10^{-2}\right)$ at $z \simeq 12.2$ plotted as a function of $\log _{10}(n / \bar{n})$. Black lines are lognormal fits to the PDFs - solid: mass-weighted PDF fit, dashed: volume-weighted PDF fit. In low density regimes, the PDFs are reasonably fit by log-normal distributions, which are known to describe turbulent flows. The disagreement at high densities is caused by the self-gravity of the gas. Right: Comparison of the $\mathrm{H}_{2}$ self-shielding factor between our integrated column density approach $\left(f_{\text {shield,column }}\right)$, a Sobolev approach (green squares), and a Jeans length approach (blue diamonds). The black line represents a one-toone mapping. As is clear, the Sobolev approximation is quite accurate for being a purely local approximation, except for a few places of stark disagreement, likely due to small velocity gradients. A combination of these two approaches would be useful for future work (e.g., [11]).

\section{Discussion and Conclusion}

Many details of this study were suppressed for space and will be presented in upcoming work. We do note several limitations and caveats, however. We neglect the photodissociation of $\mathrm{H}^{-}$, which plays a key role in $\mathrm{H}_{2}$ formation. Its impact, though, depends on the shape of the incident 
radiation spectrum and even if included would mainly shift the collapse redshift, unaffecting our results qualitatively. We also neglect the impact of large velocity gradients which, by doppler shifting of LW resonances, could cause our value of $N_{\mathrm{H}_{2}}$ to be overestimated compared with the actual column. Ref. [7], however, argue that radially coherent gas flow greatly reduces this effect's impact. A common approximation for $N_{\mathrm{H}_{2}}$ is given by $N_{\mathrm{H}_{2}}=n_{\mathrm{H}_{2}} L_{\text {char }}$ where $L_{\text {char }}$ is a characteristic length scale and $n_{\mathrm{H}_{2}}$ is the number density of molecular hydrogen. In Fig. 2(b), we compare our integrated approach for $f_{\text {shield }}^{\mathrm{H}_{2}}$ to two common approximations for $L_{\mathrm{char}}$; (1) $L_{\mathrm{char}}=L_{\mathrm{J}}$ (Jeans length), and (2), a close analogue to the Sobolev length [11], $L_{\mathrm{char}}=v_{\text {th }} /|\nabla \cdot \mathbf{v}|$, where $v_{\text {th }}=\left(k_{\mathrm{B}} T / m_{\mathrm{H}}\right)^{1 / 2}$. We note that both methods yield reasonable agreement, particularly the Sobolev length being fairly accurate over many decades in $f_{\text {shield }}^{\mathrm{H}_{2}}$. Finally, a detailed examination of HD's role is warranted, especially given suggestions (e.g., [12]) that it may play a key role in shaping the thermodynamics inside the first galaxies. We note, however, that no significant HD cooling mode was seen in the simulation.

We have explored the process of metal free gas collapse into an atomic cooling halo, focusing on the turbulent and fragmentation properties of a central, self-shielding core. We find that while the gas density PDF appears to be partly shaped by supersonic turbulence, no concurrent fragmentation takes place, at least at densities below $n=10^{8} \mathrm{~cm}^{-3}$. Additional fragmentation may take place at higher densities. Indeed, other studies have shown that fragmentation does occur in metal-free gas at high densities, even in minihalos where turbulence is thought to play a minor role.

\section{References}

[1] V. Bromm \& R. Larson, The First Stars, ARA\&A 42, 79-118

[2] E. Zackrisson et al., The Spectral Evolution of the First Galaxies. I. JWST Detection Limits and Color Criter for Pop. III Galaxies, ApJ 740, 13

[3] B. Fryxell et al., FLASH: An Adaptive Mesh Hydrodynamics Code for Modeling Astrophysical Thermonuclear Flashes, ApJS, 131, 273-334

[4] J. Johnson, T. Greif, \& V. Bromm, Occurrence of Metal-free Galaxies in the Early Universe, MNRAS 388, 26-38

[5] T. Greif, S. Glover, V. Bromm, \& R. Klessen, The First Galaxies: Chemical Enrichment, Mixing, and Star Formation, ApJ 716, 510-520

[6] B. O'Shea \& M. Norman, Pop. III Star Formation in a Lambda-CDM Universe. II. Effects of a Photodissociating Background, ApJ 673, 14-33

[7] J. Wolcott-Green, Z. Haiman, \& G. Bryan, Photodissociation of H2 in Protogalaxies: Modelling Self-Shielding in Three-Dimensional Simulations, MNRAS 418, 838-852

[8] K. Omukai, Primordial Star Formation under Far-Ultraviolet Radiation, ApJ 546, 635

[9] C. Federrath, R. Banerjee, P. Clark, \& R. Klessen, Modeling Collapse and Accretion in Turbulent Gas Clouds: Implementation and Comparison of Sink Particles in AMR and SPH, ApJ 713, 269-290

[10] A. Kritsuk, M. Norman, P. Padoan, \& R. Wagner, The Statistics of Supersonic Isothermal Turbulence, ApJ 665, 416-431

[11] P. Clark et al., Gravitational Fragmentation in Turbulent Primordial Gas and the Initial Mass Function of Pop. III Stars, ApJ 727, 110 
[12] J. Johnson \& V. Bromm, The Cooling of Shock-Compressed Primordial Gas, MNRAS 366, 247-256 